Elsevier Scientific Publishers Ireland, Ltd.

CELDIF 00645

ir

\title{
Maintenance of compaction and adherent-type junctions in mouse morula-stage embryos
}

\author{
Ilkka Rẹima \\ Deparsmenis of Pathology' and Electron Microscopy, University of Helsinki, Helsinki, Finland
}

(Accepted 22 November 1989)

\section{(}

Timed morulae of different stages of development were exposed to cytochalasin B causing depolymerisation of microfilaments and to ECCD-1 antibodies interacting with $\mathrm{Ca}^{2+}$-dependent adhesion molecules or cultured in the absence of calcium. All three treatments decompacted mid-morula-stage embryos within one hour. Late morulae were resistant to ECCD-1 antibody treatment and relatively resistant to calcium-free cultivation, but not to cytochalasin B treatment. Scanning electron microscopy revealed that the decompacting treatments not only loosened the interblastomere contacts but also resulted in rearrangement of the cell surface microvilli. Transmission electron microscopy showed that normal, untreated embryos had specialized membrane junctions in the most apical regions of the interblastomere contacts. Immunoelectron microscopy revealed that these apical junction areas contained vinculin, a protein typical of adherent junctions. Upon decompaction the apical junctions disappeared completely. When transferred back to the normal medium, the embryos rapidly started to recompact. Simultaneously the apical junctions and cell surface microvilli reassumed the organization characteristic of the morula stage. Late morulae that were resistant to treatment had normal apical junctional areas. During subcultivation in the normal medium, the treated morulae developed into morphologically normal blastocysts. These data indicate that adherent-type junctions and cell surface microvilli participate in the initiation and maintenance of compaction of morula-stage embryos.

\section{(}

\section{Introduction}

Cell-cell interactions in morula-stage embryos are important for the normal development of the blastocyst and lead to the differentiation of the inner cell mass (ICM) and the trophectoderm (Tarkowski and Wroblew'ska, 1967; Hillman et al., 1972; Kelly, 1977; Graham and Lehtonen, 1979).

Correspondence address: I. Reima, Department of Pathology, University of Helsinki, Haartmaninkatu 3, SF-00290 Helsinki, Finland.
These interactions depend on intercellular contacts. During embryogenesis, the formation of specialized intercellular junctions begins at the time of compaction during which the blastomeres of the 8-cell embryo flatten against each other and maximize their areas of intercellular contacts (Lewis and Wright, 1935; Ducibella and Anderson, 1975; Lehtonen, 1980). At the same time changes occur in the distribution of surface microvilli as the blastomeres start to polarize (Ducibella et al., 1977; Ziomek and Johnson, 1980; Johnson et al., 1986b).

The compaction can be manipulated by using

0922-3371/90/\$03.50@ 1990 Elsevier Scientific Publishers Ireland, Ltd. 